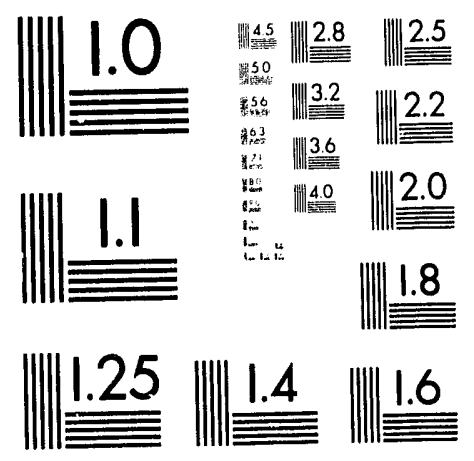



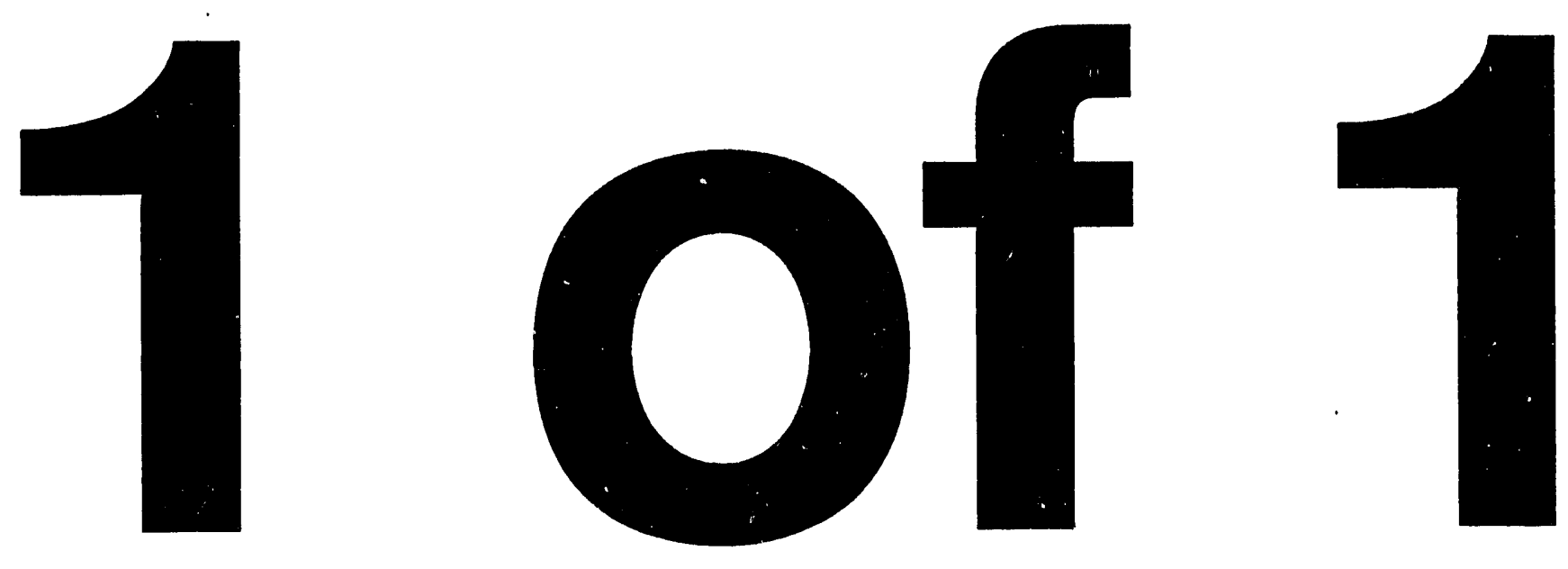


$$
\begin{aligned}
& \text { PPPL- L'FP-296.4 } \\
& \text { Conf } 931070--3
\end{aligned}
$$

\title{
NON-LOCAL TRANSPORT IN A TOKAMAK PLASMA DIVERTOR WITH RECYCLING
}

\author{
Z. A BOU-ASSALEH, M. PETRA VIC, R. VESEY \\ Plasma Physics Laboratory, Princeton University \\ Princeton, New Jersey 08543, USA.
}

\section{J.P. MATTE, T.W. JOHNSTON}

INRS-Énergie et Matériaux

1650 Montée Ste-julie, Varennes, Québec J3X 1S2, CANADA.

\section{Introduction}

The plasma transport, particle and energy fluxes, near the divertor plate with high recycling has been modeled by using an electron kinetic code (Fokker-Planck International) ${ }^{1,2,3}$, in conjunction with a two-fluid ambipolar code ${ }^{23}$. We include the effects of ionisation and excitation of the hydrogen atoms. The electron energy distribution calculated from the kinetic code shows a large deviation from Maxwellian especially near the plate. This deviation from Maxwellian is due to the non-local transport of the suprathermal electrons from the SOL, and due also to the absorption of the fast electrons by the target plate. The heat flux near the plate is shown to be nonlocal, in that it is not determined uniquely by the local plasma parameters. Therefore the classical transport coefficients in the fluid model must be modified by including a nonlocal effect to produce the kinetic results. The kinetic calculation is compared with those of the fluid code with different values of the electron heat flux limiter factor (f). To reduce the computer load, the initial condition we used corresponds to the equilibrium solution already found with the fluid code with $\mathrm{f}=0.2$. The fluid and Fokker-Planck codes are relaxed until all transients associated with electron dynamics have disappeared.

In section II, we present the kinetic code. The fluid code is presented in section III. The boundary conditions used in these simulations are given in section IV. Finally the results and conclusion of these simulations are presented in section $\mathrm{V}$.

\section{1D-2Fluid Code}

The one-dimensional, ambipolar two-fluid time dependent code ${ }^{2.3}$ including ion viscosity and heat flow has been used to study the plasma transport in a divertor tokamak. The basic equations and transport coefficients used in this code are taken from Braginskii [5]. In our fluid model we limit the electron conduction heat flux by using the harmonic mean between the Spitzer-Härm and the flux-limited fluxes [6]:

$$
Q_{c \text { cull }}^{\leftarrow}=q_{S h}\left[1+\left|q_{S H}\right| \overline{f_{n} T_{e} v_{e}}\right]^{-1}
$$

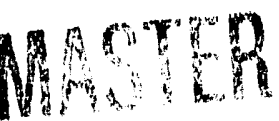


where $v_{e}=\sqrt{T_{e} / m e}, m_{e}$ is the mass of the electron and $f$ is the so called flux limiter.

\section{Fokker-Planck International Code:}

The Fokker-Planck International (FPI) $\operatorname{code}^{1,2,3}$ is one-dimensional in configuration space, but two-dimensional in velocity space (azimuthal symmetry is assumed). In this code electrons are treated with a kinetic model but the ions and the neutral particles are treated as fluids. The FPI code advances the Vlasov-Fokker-Planck equation for the electron distribution function in time. Thus the kinetic equation for the electrons is given by :

$$
\frac{\partial f}{\partial t}+v_{x} \frac{\partial f}{\partial x}+\frac{e E}{m_{e}} \frac{\partial f}{\partial v_{x}}=\left(\frac{\partial f}{\partial t}\right)_{(e-i, e-e)}+\left(\frac{\partial f}{\partial t}\right)_{\left(E_{e i}\right)}+\left(\frac{\partial f}{\partial t}\right)_{(e-n)}
$$

The second and third terms in the left hand side of Eq.(2) are the advection and the acceleration due to the electric field, respectively. The right-hand side terms represent respectively electron-ion and electron-electron Coulomb scattering, electron-ion energy exchange and inelastic electron-neutral collisions (ionization and excitation).

\section{Boundary Conditions}

\section{1D-2Fluid Code:}

At the left boundary we fixed the electron and ion temperatures. The particle flux at this boundary is null. At the neutralizer plate, we assume that the incoming plasma is absorbed, and the drift velocity is assumed to be equal to or higher than the ion sound velocity, i.e,

$$
v \geq c_{s}=\sqrt{\left(T_{e}+T_{i}\right) / m_{i}}
$$

The ion and electron heat flux at the plate are taken as:

$$
Q_{1}=3.5 T_{i} n V ; Q_{e}=6 T_{e} n V
$$

The boundary condition at the plate for the neutral density is calculated by assuming that the neutral flux at the plate is given by:

$$
n_{n} v_{n} \sin \theta=R n_{i} v_{i}
$$

where $\theta$ is the angle between the plate and the magnetic field lines, we used $\theta=5^{\circ}$. Here we have assumed that the recycling factor is $R=0.9$. For the neutral velocity $v_{n}$, we assume $\frac{1}{2} m_{i} v_{n}^{2}=3 \mathrm{eV}$, corresponding to the Frank-Condon energy. 


\section{Electron Kinetic code (FPI)}

Plasma is assur $u$ to he flowing in from the left, $x=0$, with given plasma density $n_{0}$, drift velocity $v_{0}$, and electron and ion temperatures $\left(T_{e}=T_{1}=T_{0}\right)$. The electron distribution function on the left is adjusted in accordance with the hot wall technique explained in reference [1]. The neutralizer plate at the right boundary $(x=L)$ is modelled as follows: all incident ions are absorbed by the plate, incoming electrons with a longitudinal velocity above $v_{R}$ are absorbed as well and others are reflected. The velocity $v_{R}$ is adjusted so as to give a zero net current at the plate. This boundary condition removes the need to simulate the sheath itself. The ion and neutral dynamics are the same in the electron kinetic code as in the two-fluid code.

\section{Results and Conclusions:}

The equilibrium fluid results of the plasma with high recycling $(R=0.9)$ and $(f=0.2)$, near the divertor plate are presented in Figs. (1) and (2). In Fig. (1) we plot the profiles of the plasma and neutral densities, electron and ion temperatures $\left(T_{e}, T_{i}\right)$. The profiles of the plasma particle flux $\left(n_{i} V_{i}\right)$, ionisation and excitation rates $\left(S_{i o n}, S_{\text {exc }}\right)$ are plotted in Fig. (2).

This equilibrium solution is used as initial condition for the kinetic code and for the fluid code runs preformed with a range of heat flux limiter factors, f. Results from the kinetic and fluid calculations (with different values of $f$ ) are presented in Figs.(3) and (4).

In Fig.(3) we plot the electron temperature profiles which were calculated by the kinetic and fluid codes. The values of the electron heat flux limiter factor $f$ used in the fluid model are $0.1,0.2,0.6$, and $\infty$ (Spitzer). It is clear from this figure that for no value of the flux limiter do the fluid calculations of the electron temperature agree with the kinetic calculations. To understand the discrepancy more clearly we therefore examine the profiles of the electron conduction heat flux calculated from the kinetic and fluid codes, see Fig.(4). In Fig. (4) we also plotted the profile of $q_{e}$ calculated from the FPI profile of $T_{e}$, by using the classical formula, Eq. (1), with $f=0.2$. This calculation disagrees significantly with the kinetic and fluid calculations, and the discrepancy is worse if a value of the flux limiter $f$ different from 0.2 is used. From this we see that the fluid calculation does not agree with the kinetic calculation for any value of the heat flux limiter and this is due to the non-local effect on the electron transport in the SOL. The classical coefficient of the electron heat flux must be modified everywhere in the system in order to reproduce the kinetic results. In our model we did this modification numerically as follows: in the fluid code we use the Te profile which is calculated from the kinetic code and also force the local electron conducting heat flux, $q_{e}$, to agree with that of the kinetic model by multiplying by the following correction factors:

$$
C F Q=q_{t-K i n e t \text { ic }} / q_{\text {e-Fluid }}\left(T_{\text {e-kinetic }}\right)
$$

In Fig.(5) we present the correction factor in the fluid code for the electron heat flux for 
this particular simulation.

\section{Acknowledgement}

Work supported by Canadian National Fusion program, and by the U.S. Department of Energy Contract No.DE-AC(12-76-CHO-3073.

\section{References}

1. J. H. Rogers, J. S. De Groot, Z. Abou-Assaleh, J. P. Matte, T. W. Johnston, and M. D. Rosen, Phys. Fluids B 1, (1989), 741-749.

2. Z. Abou-Assaleh, Ph.D. Desseration, 1991. Université du Québec,INRS-Énergie et Matériaux, Varennes, Québec, CANADA.

3. Z. Abou-Assaleh, J.P. Mutte, T. W. Johnston and R. Marchand. Contrib. Plasma Phys. 32 (1992) 3/4, 268-272.

4. Yu. L. Igitkhanov and A. M. Runov. Contrib. Plasma Phys. 32 (1992) 3/4, 308-313.

5. S.I. Braginskii, in: Reviews of plasma physics, Vol. 1, Ed. M. A. Leontvich (Consultants Bureau, New York, 1965), p. 205.

6. L. Spitzer and R. Härm, Phy. Rev. 89, 977 (1953).

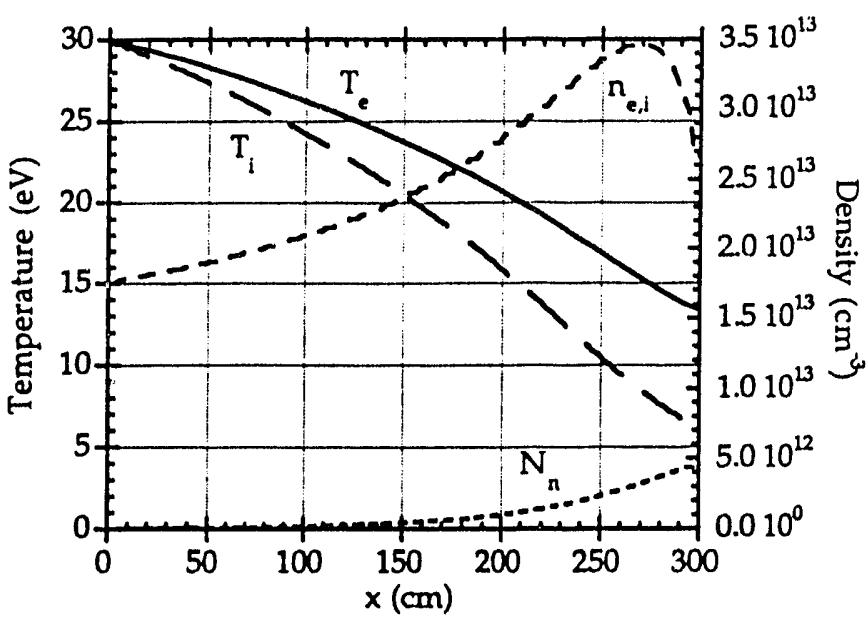

Figure 1 Fluid equilibrium solution of edge plasma near the plate with recycling $R=0.9$ and $t=0.2$ : Profiles of $T_{e}, T_{1}, n_{i e}$, and $n_{n}$.

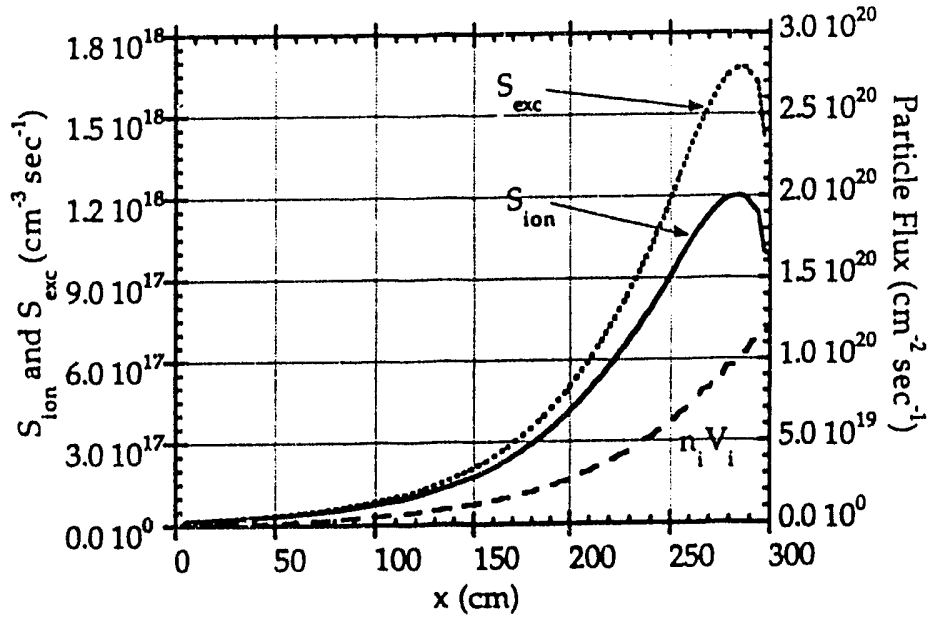

Figure 2 Fluid equilibrium solution of edge plasma near the plate with recycling $R=0.9$ and $f=0.2$ : Profiles of $n_{1} v_{i}, S_{e x c}$, and $S_{\text {ioni. }}$. 


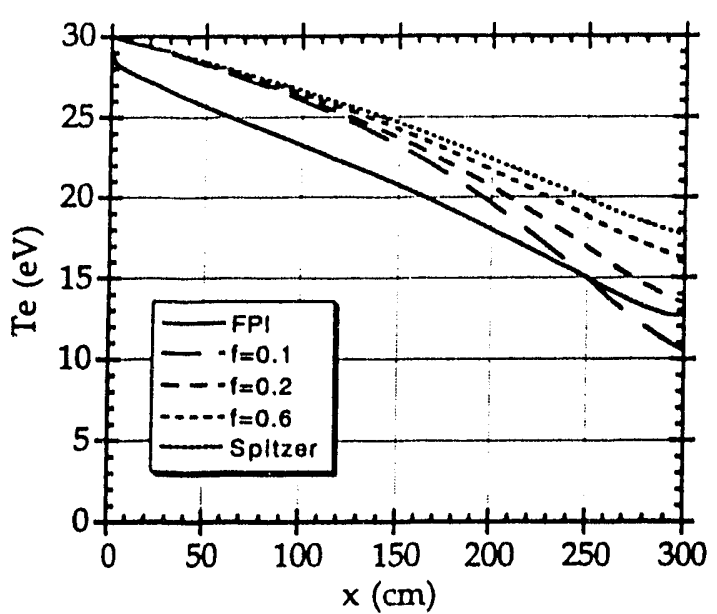

Figure 3 Fluid and Fokker-Pliunck simulations: Profiles of $T_{e}$ from Fokker-Planck, and fluid calculations with $f=0.1,0.2,0.6,1$ (Spizer).

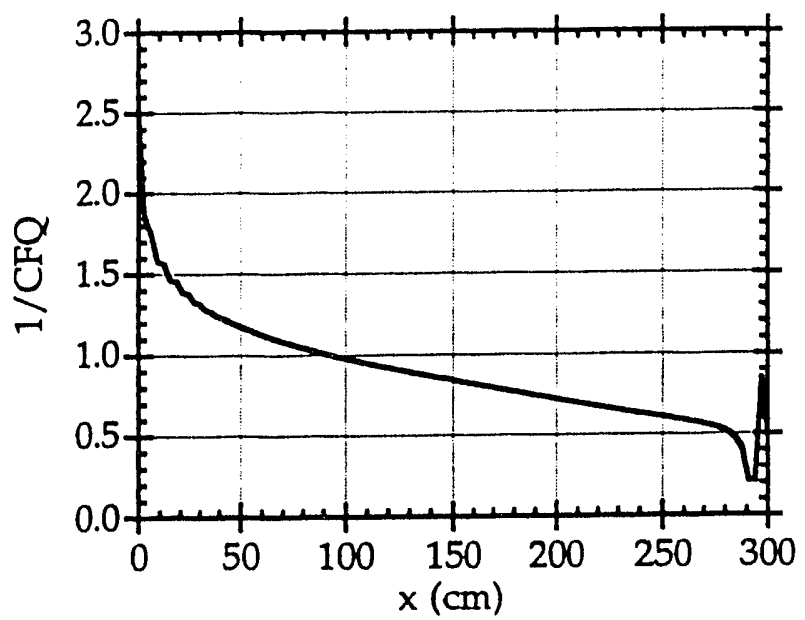

Figure 5 Profile of the electron conduction heat flux correction factor (CFQ).

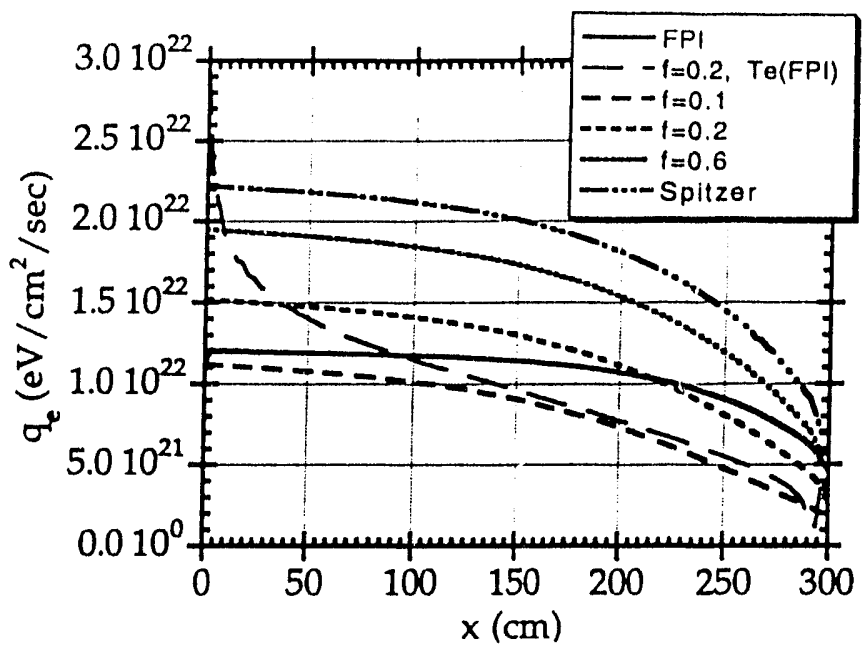

Figure 4 Fluid and Fokker-Planck simulations: Profiles of $\mathrm{q}_{e}$ from Fokker-Planck, and fluid calculations with $f=0.1,0.2,0.6, \mathrm{l}$ (Spitzer). 


\title{
NON-LOCAL TRANSPORT IN A TOKAMAK PLASMA DIVERTOR WITH RECYCLING
}

\author{
Z. ABOU-ASSALEH, M. PETRA VIC, R. VESEY \\ Plasma Physics Lahoratory, Princeton University \\ Princeton, New Jersey 08543, USA. \\ J.P. MATTE, T.W. JOHNSTON \\ INRS-Énergie et Matériaux \\ 1650 Montée Ste-julie, Varennes, Québec J3X 1S2, CANADA.
}

\section{Introduction}

The plasma transport, particle and energy fluxes, near the divertor plate with high recycling has been modeled by using an electron kinetic code (Fokker-Planck International) ${ }^{1,2,3}$, in conjunction with a two-fluid ambipolar code ${ }^{23}$. We include the effects of ionisation and excitation of the hydrogen atoms. The electron energy distribution calculated from the kinetic code shows a large deviation from Maxwellian especially near the plate. This deviation from Maxwellian is due to the non-local transport of the suprathermal electrons from the SOL, and due also to the absorption of the fast electrons by the target plate. The heat flux near the plate is shown to be nonlocal, in that it is not determined uniquely by the local plasma parameters. Therefore the classical transport coefficients in the fluid model must be modified by including a nonlocal effect to produce the kinetic results. The kinetic calculation is compared with those of the fluid code with different values of the electron heat flux limiter factor ( $f$ ). To reduce the computer load, the initial condition we used corresponds to the equilibrium solution already found with the fluid code with $\mathrm{f}=0.2$. The fluid and Fokker-Planck codes are relaxed until all transients associated with electron dynamics have disappeared.

In section II, we present the kinetic code. The fluid code is presented in section III. The boundary conditions used in these simulations are given in section IV. Finally the results and conclusion of these simulations are presented in section $\mathrm{V}$.

\section{1D-2Fluid Code}

The one-dimensional, ambipolar two-fluid time dependent code ${ }^{2,3}$ including ion viscosity and heat flow has been used to study the plasma transport in a divertor tokamak. The basic equations and transport coefticients used in this code are taken from Braginskii [5]. In our fluid model we limit the electron conduction heat flux by using the harmonic mean between the Spitzer-Härm and the flux-limited fluxes [6]:

$$
\left.Q_{r(H)}=q_{S H} 1+\left|q_{S H}\right| \overline{f n T_{e} v_{e}}\right]^{-1}
$$


where $v_{e}=\sqrt{T_{e} / m e}, m_{e}$ is the mass of the electron and $E$ is the so called flux limiter.

\section{Fokker-Planck International Code:}

The Fokker-Planck International (FPI) $\operatorname{code}^{1.2,3}$ is one-dimensional in configuration space, but two-dimensional in velocity space (azimuthal symmetry is assumed). In this code electrons are treated with a kinetic model but the ions and the neutral particles are treated as fluids. The FPI code advances the Vlasov-Fokker-Planck equation for the electron distribution function in tinie. Thus the kinetic equation for the electrons is given by :

$$
\frac{\partial f}{\partial t}+v_{x} \frac{\partial f}{\partial x}+\frac{e E}{m_{e}} \frac{\partial f}{\partial v_{x}}=\left(\frac{\partial f}{\partial t}\right)_{(e-i, e-e)}+\left(\frac{\partial f}{\partial t}\right)_{\left(E_{e i}\right)}+\left(\frac{\partial f}{\partial t}\right)_{(e-n)}
$$

The second and third terms in the left hand side of Eq.(2) are the advection and the acceleration due to the electric field, respectively. The right-hand side terms represent respectively electron-ion and electron-electron Coulomb scattering, electron-ion energy exchange and inelastic electron-neutral collisions (ionization and excitation).

\section{Boundary Conditions}

\section{1D-2Fluid Code:}

At the left boundary we fixed the electron and ion temperatures. The particle flux at this boundary is null. At the neutralizer plate, we assume that the incoming plasma is absorbed, and the drift velocity is assumed to be equal to or higher than the ion sound velocity, i.e,

$$
v \geq c_{s}=\sqrt{\left(T_{e}+T_{i}\right) / m_{i}}
$$

The ion and electron heat flux at the plate are taken as:

$$
Q_{1}=3.5 T_{1} n V ; Q_{e}=6 T_{e} n V
$$

The boundary condition at the plate for the neutral density is calculated by assuming that the neutral flux at the plate is given by:

$$
n_{n} v_{n} \sin \theta=R n_{i} v_{i}
$$

where $\theta$ is the angle between the plate and the magnetic field lines, we used $\theta=5^{\circ}$. Here we have assumed that the recycling factor is $R=0.9$. For the neutral velocity $v_{n}$, we assume $\frac{1}{2} m_{i} v_{n}^{2}=3 \mathrm{eV}$, corresponding to the Frank-Condon energy. 


\section{Electron Kinetic code (FPI)}

Plasma is assumed $t o$ he flowing in fiom the left, $x=0$, with given plasma density $n_{0}$, drift velocity $v_{0}$, and electron and ion temperatures $\left(T_{e}=T_{i}=T_{0}\right)$. The electron distribution function on the left is adjusted in accordance with the hot wall technique explained in reference [1]. The neutralizer plate at the right houndary $(x=L)$ is modelled as follows: all incident ions are absorbed by the plate, incoming electrons with a longitudinal velocity above $v_{R}$ are absorbed as well and others are reflected. The velocity $v_{R}$ is adjusted so as to give a zero net current at the plate. This boundary condition removes the need to simulate the sheath itself. The ion and neutral dynamics are the same in the electron kinetic code as in the two-fluid code.

\section{Results and Conclusions:}

The equilibrium fluid results of the plasma with high recycling $(R=0.9)$ and $(f=0.2)$, near the divertor plate are presented in Figs. (1) and (2). In Fig. (1) we plot the profiles of the plasma and neutral densities, electron and ion temperatures $\left(T_{e}, T_{1}\right)$. The profiles of the plasma particle flux $\left(n_{i} v_{1}\right)$, ionisation and excitation rates $\left(S_{10 n^{\prime}} S_{e x c}\right)$ are plotted in Fig. (2).

This equilibrium solution is used as initial condition for the kinetic code and for the fluid code runs preformed with a range of heat flux limiter factors, $f$. Results from the kinetic and fluid calculations (with different values of $f$ ) are presented in Figs.(3) and (4).

In Fig.(3) we plot the electron temperature profiles which were calculated by the kinetic and fluid codes. The values of the electron heat flux limiter factor $f$ used in the fluid model are $0.1,0.2,0.6$, and $\infty$ (Spitzer). It is clear from this figure that for no value of the flux limiter do the fluid calculations of the electron temperature agree with the kinetic calculations. To understand the discrepancy more clearly we therefore examine the profiles of the electron conduction heat flux calculated from the kinetic and fluid codes, see Fig.(4). In Fig. (4) we also plotted the profile of $q_{e}$ calculated from the FPI profile of $T_{e}$, by using the classical formula, Eq. (1), with $f=0.2$. This calculation disagrees significantly with the kinetic and fluid calculations, and the discrepancy is worse if a value of the flux limiter $f$ different from 0.2 is used. From this we see that the fluid calculation does not agree with the kinetic calculation for any value of the heat flux limiter and this is due to the non-local effect on the electron transport in the SOL. The classical coefficient of the electron heat flux must he modified everywhere in the system in order to reproduce the kinetic results. In our model we did this modification numerically as follows: in the fluid code we use the Te profile which is calculated from the kinetic code and also force the local electron conducting heat flux, $\mathrm{q}_{e}$, to agree with that of the kinetic model by multiplying by the following correction factors:

$$
C F Q=q_{\text {-K K } 1 \text { net } 1 C^{\prime}} / q_{\text {e-Fluid }}\left(T_{\text {e-kinetic }}\right)
$$

In Fig.(5) we present the correction factor in the fluid code for the electron heat flux for 
this particular simulation.

\section{Acknowledgement}

Work supported by Canadian National Fusion program, and by the U.S. Department of Energy Contract No.DE-AC()2-76-CHO-3073.

\section{References}

1. J. H. Rogers, J. S. De Groot, Z. Abou-Assaleh, J. P. Matte, T. W. Johnston, and M. D. Rosen, Phys. Fluids B 1, (1989), 741-749.

2. Z. Abou-Assaleh, Ph.D. Desseration, 1991. Université du Québec,INRS-Énergie et Matériaux, Varennes, Québec, CANADA.

3. Z. Abou-Assaleh, J.P. Matte, T. W. Johnston and R. Marchand. Contrib. Plasma Phys. 32 (1992) 3/4, 268-272.

4. Yu. L. Igitkhanov and A. M. Runov. Contrib. Plasma Phys. 32 (1992) 3/4, 308-313.

5. S.I. Braginskii, in: Reviews of plasma physics, Vol. 1, Ed. M. A. Leontvich (Consultants Bureau, New York, 1965), p. 205.

6. L. Spitzer and R. Härm, Phy. Rev. 89, 977 (1953).

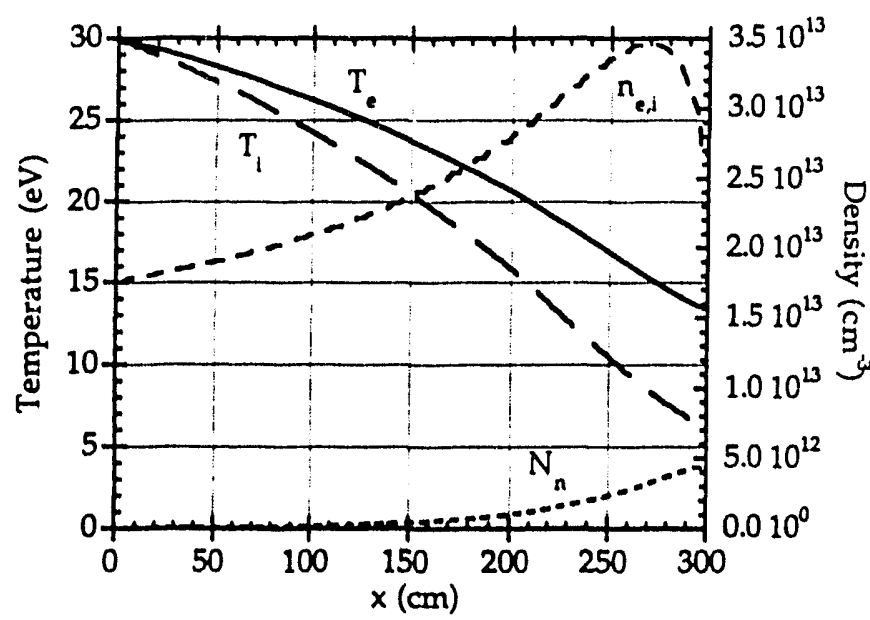

Figure 1 Fluid equilibrium solution of edge plasma near the plate with recycling $\mathrm{R}=0.9$ and $\mathrm{f}=0.2$ : Profiles of $T_{e}, T_{i}, n_{l e}$, and $n_{p}$.

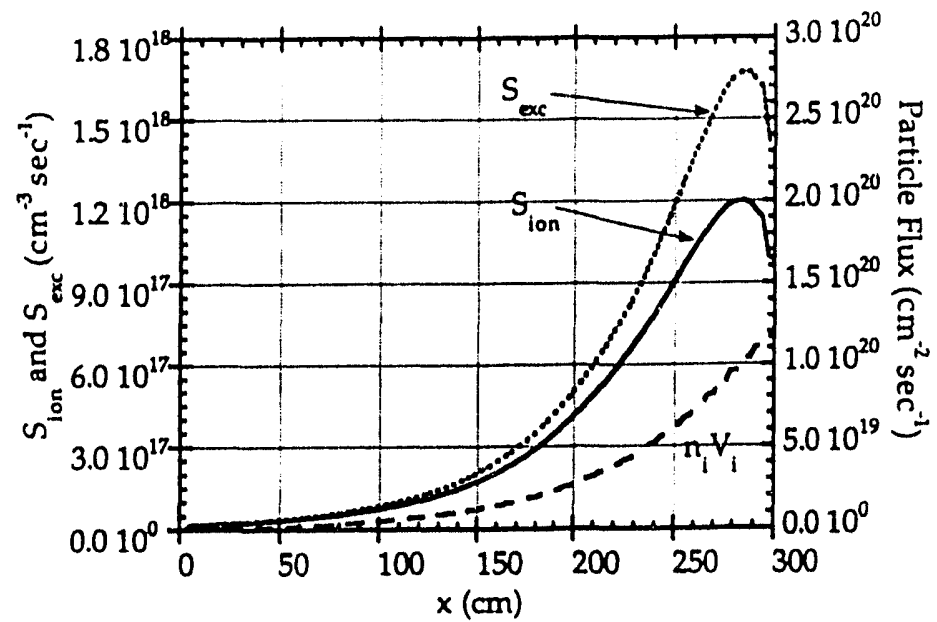

Figure 2 Fluid equilibrium solution of edge plasma near the plate with recycling $R=0.9$ and $f=0.2$ : Profiles of $n_{1} v_{i}, S_{\text {exc. }}$, and $S_{\text {ioni }}$. 


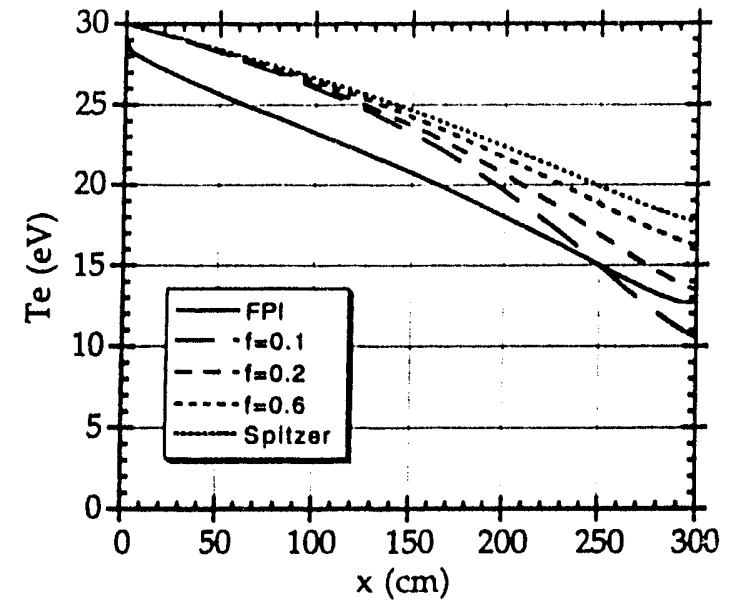

Figure 3 Fluid and Fokker-Pliunck simulations: Profiles of $T_{e}$ from Fokker-Planck, and fluid calculations with $f=0.1,0.2,0.6, \mathrm{l}$ (Spitzer).

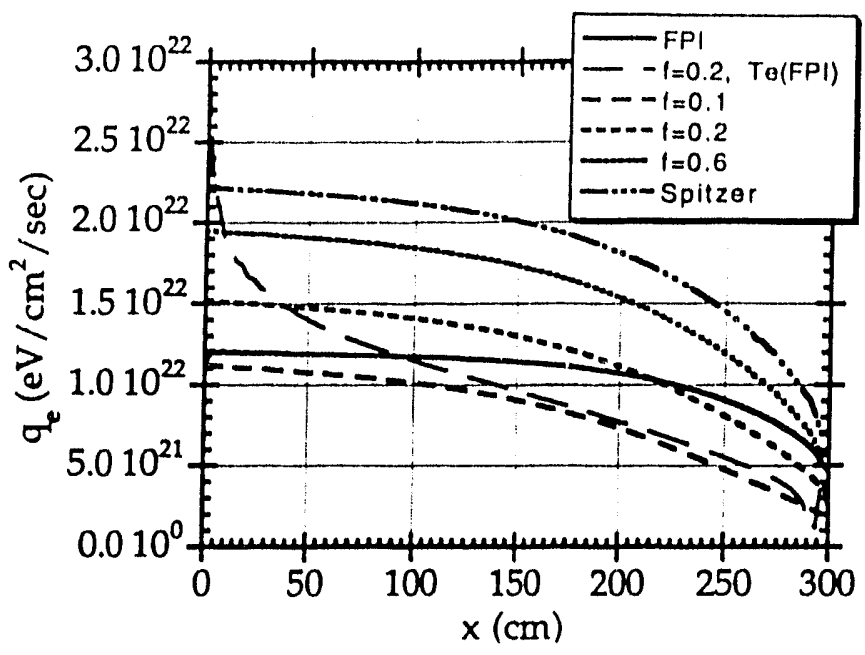

Figure 4 Fluid and Fokker-Planck simulations: Profiles of $q_{e}$ from Fokker-Planck, and fluid calculations with $\mathrm{f}=0.1,0.2,0.6, \mathrm{l}$ (Spitzer).

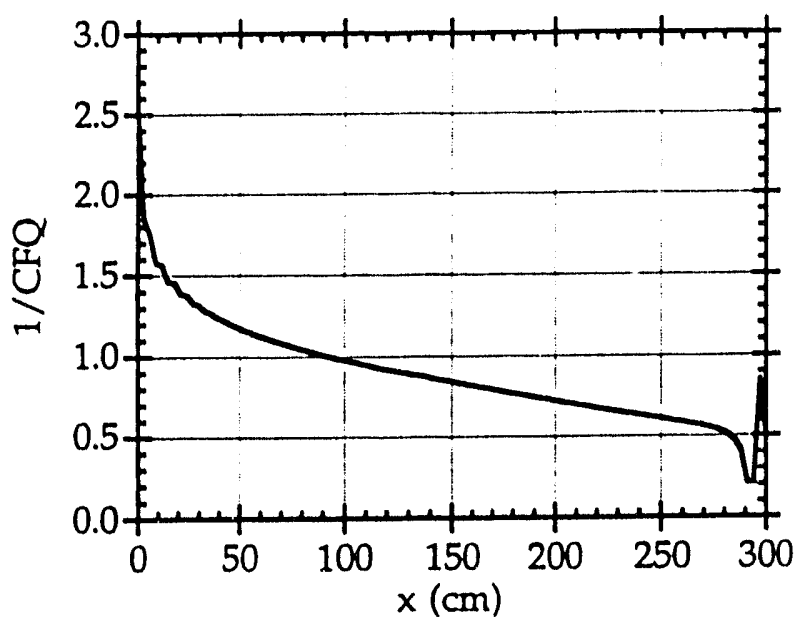

Figure 5 Profile of the electron conduction heat flux correction factor (CFQ). 

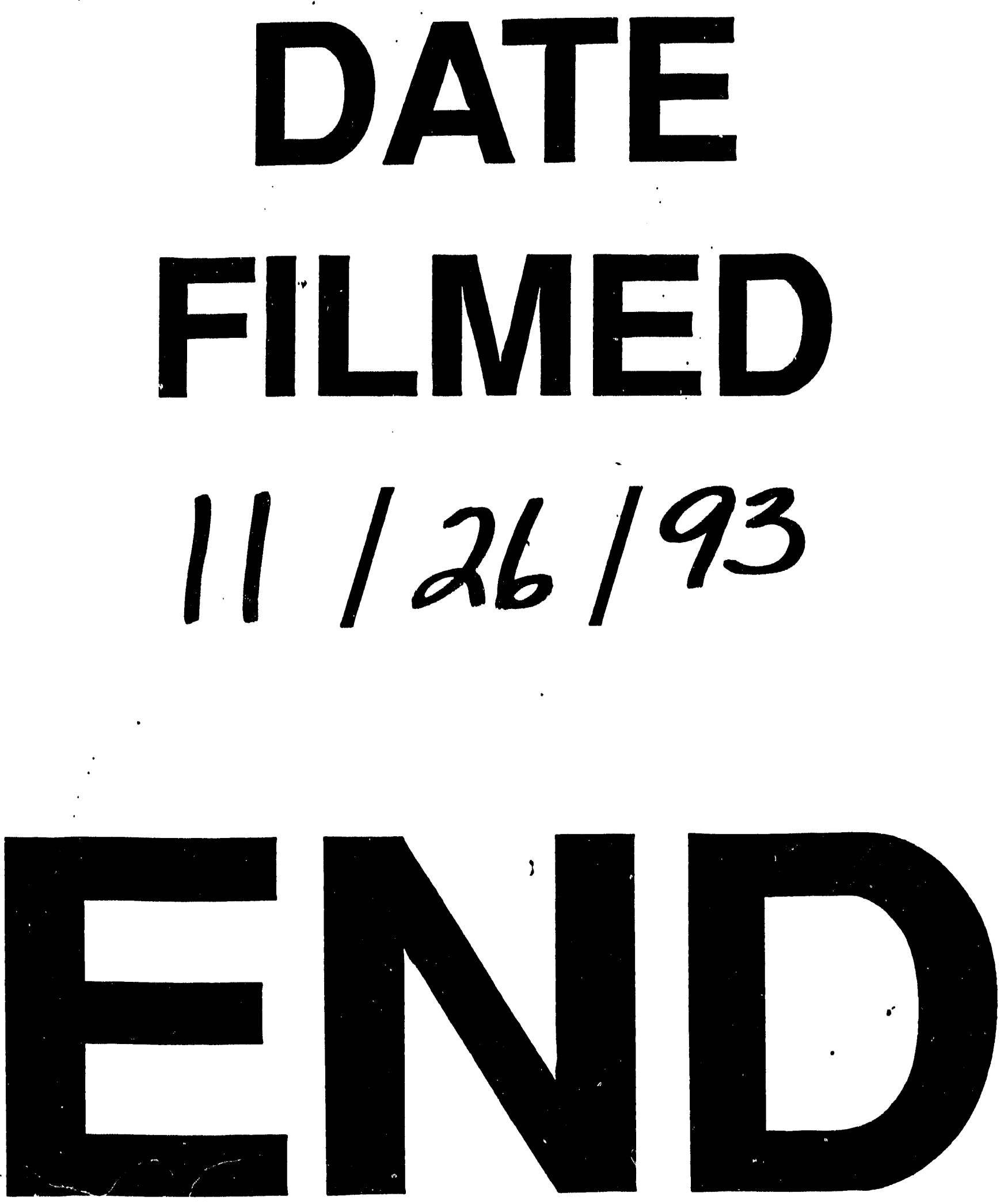
